\title{
MicroRNA let-7 regulates the expression of ecdysteroid receptor (ECR) in Hyalomma asiaticum (Acari: Ixodidae) ticks
}

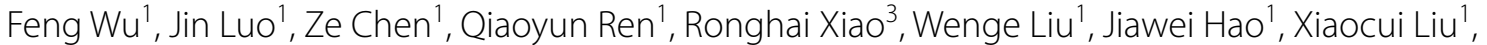 \\ Junhui Guo ${ }^{1,2}$, Zhiqiang Qu ${ }^{1,2}$, Zegong Wu' ${ }^{1}$, Hui Wang ${ }^{1,5}$, Jianxun Luo ${ }^{1}$, Hong Yin ${ }^{1,4}$ and Guangyuan Liu ${ }^{*}$
}

\begin{abstract}
Background: Ticks are blood-sucking arthropods that can transmit diseases to humans and animals. These arthropods are the second most important vectors of pathogens. MicroRNAs are a class of conserved small noncoding RNAs that play regulatory roles in gene expression at the post-transcriptional level. Molting is an important biological process in arthropods. Research on the molting process is important for understanding tick physiology and control.

Methods: Dual-luciferase reporter assays were used to assess the role of miRNA let-7 in ecdysteroid receptor (ECR) biology. The expression levels of ECR and let-7 were measured by real-time qPCR before and after tick molting. To explore the function of let-7 and ECR, we performed overexpression and knocking down of let-7 and RNAi of ECR in tick nymphs. The biological function of let-7 in molting was explored by injecting nymphs, ten days after engorgement, with let-7 agomir for overexpression and let-7 antagomir for knocking down. The rate of molting was then determined. ECR dsRNA was injected into ticks to evaluate the function of ECR by gene silencing. The expression of ECR and let-7 was measured using RT-qPCR. All data were analyzed using GraphPad Prism v.6.
\end{abstract}

Results: The results of the luciferase assay using a eukaryotic expression system revealed that ECR was a natural target of let-7. Let-7 overexpressed by agomir affected the rate of molting $(P<0.01)$ and the period of molting $(P<0.01)$. Let-7 antagomir for knockdown affected the period of molting $(P<0.01)$, but there was no effect on the rate of molting $(P=0.27)$. ECR dsRNA gene silencing significantly affected the rate of molting $(P<0.05)$.

Conclusions: This study demonstrated that let-7 can regulate the expression of ECR and that let-7 can affect molting in ticks. Our results help to understand the regulation of let-7 by 20-hydroxyecdysone (20E) and will provide a reference for functional analysis studies of microRNAs in ticks.

Keywords: Hyalomma asiaticum, microRNA let-7, Ecdysone receptor, 20-hydroxyecdysone, Molting regulation

\section{Background}

Ticks are blood-sucking arthropods that secrete immunomodulatory molecules to antagonize the inflammatory and immune responses of the host $[1,2]$. Ticks are the second most important vectors of bacteria, viruses, parasites and other pathogens [3]. Medically important

\footnotetext{
*Correspondence: liuguangyuan@caas.cn

${ }^{1}$ State Key Laboratory of Veterinary Etiological Biology, Key Laboratory of Veterinary Parasitology of Gansu Province, Lanzhou Veterinary Research Institute, Chinese Academy of Agricultural Science, Xujiaping 1, Lanzhou 730046, Gansu, People's Republic of China

Full list of author information is available at the end of the article
}

tick-borne diseases, including tick-borne encephalitis, granulocytic ehrlichiosis, babesiosis and Lyme disease, have a huge impact on human health $[4,5]$.

MicroRNAs (miRNAs) are a class of small non-coding RNAs with a length of 21-24 nucleotides. These molecules are involved in the post-transcriptional regulation of mRNAs in plants and animals [6,7]. There are many miRNAs in multicellular organisms, and some are evolutionarily conserved [8]. Let-7 is the second miRNA identified in Caenorhabditis elegans and is evolutionally conserved in bilateral animals [6,9]. This molecule is essential in the heterochrony pathway, playing a role in 
the regulation of developmental events [10]. In Drosophila, let-7 has been implicated in developmental timing [10, 11], wing development [12] and neurogenesis [13]. It also participates in innate immunity by targeting the antimicrobial peptide diptericin [14].

Let-7 is conserved in several species. The expression of four miRNAs, mir-34, mir-100, mir-125 and let-7, undergo temporal changes at larval-to-pupal or pupalto-adult stages, and respond to ECD (steroid 20-hydroxyecdysone) and/or JH (juvenile hormone) in S2 cells of Drosophila [15]. In a study of silk worms [16], let-7 was shown to regulate molting and metamorphosis. Based on these reports, we hypothesized that let-7 could regulate molting and metamorphosis in Hyalomma asiaticum.

20-hydroxyecdysone (20E), a ubiquitous steroid hormone, coordinates multiple developmental events by recruiting the complex 20E-ECR/USP (ultraspiracle protein, USP), which directly activates the expression of many early-response genes, including E74, E75 and Broad-Complex. In turn, the products of early-response genes trigger the expression of late-response genes [1719]. Ecdysone triggers molting and metamorphosis in insects. During metamorphosis, most cells are regulated by hormones, and cells from different tissues are regulated by different developmental pathways. In addition, ecdysone plays an essential role in oogenesis [20]. In a study of Drosophila, 20E-induced broad-complex could regulate miRNAs (let-7, miR-100, miR-34 and miR-125) which are involved in the 20E signaling pathway [21]. The present study shows that let-7 can regulate expression of ECR and molting in $H$. Asiaticum and provides an insight into the regulation of let-7 by 20 -hydroxyecdysone (20E). The study may also serve as a reference for future research on the function of tick miRNA.

\section{Methods}

\section{Tick collection}

Hyalomma asiaticum ticks were collected from Gansu Province and have been maintained in our laboratory since 2006. Ticks were reared by feeding on rabbits for generations in the laboratory. All tick developmental stages were maintained at a temperature of $30 \pm 2{ }^{\circ} \mathrm{C}$ and relative humidity of $80 \pm 5 \%$ [22].

\section{Amplification of the $3^{\prime}$-UTR region of ECR}

Total RNA was isolated from partially-engorged adult female ticks using Trizol reagent (TaKaRa, Shiga, Japan). First-strand cDNAs were synthesized using the protocol for transcriptase XL (Avian Myeloblastosis Virus, AMV) (TaKaRa) and oligo dT18. A 3'-RACE cDNA amplification kit (Invitrogen, California, USA) was used to obtain the $3^{\prime}$-sequences of the ECR genes of $H$. asiaticum. The gene-specific primers GSP-ECR-3UTR-F1 and GSP-ECR-3UTR-F2 were used in $3^{\prime}$-RACE. Nested PCR was performed to amplify the sequence of $3^{\prime}$-UTR. The first PCR was performed using primers GSP-ECR3UTR-F1 and AUAP (universal amplification primer) by denaturing at $95{ }^{\circ} \mathrm{C}$ for 3 min followed by 35 cycles at $95{ }^{\circ} \mathrm{C}$ for $30 \mathrm{~s}, 55^{\circ} \mathrm{C}$ for $30 \mathrm{~s}$ and $72{ }^{\circ} \mathrm{C}$ for $90 \mathrm{~s}$, and a final extension step at $72{ }^{\circ} \mathrm{C}$ for $10 \mathrm{~min}$. Nested PCR was performed using the first-round PCR product (diluted 100 times in RNase-free water) as a template and primers AUAP and GSP-ECR-3UTR-F2. The PCR product was purified and cloned into the PMD 19-T vector (TaKaRa). Gene sequencing was performed by GenScript (Nanjing, China).

\section{Cloning of let-7}

The primers and loop primer were designed according to the mature sequence of isc-let-7 (MIMAT0012680, miRBase). The loop primer (5'-GTC GTA TCC AGT GCA GGG TCC GAG GTA TTC GCA CTG GAT ACG ACA CTA TAC A-3') was used to synthesize the first-strand cDNA. PCR was performed using let-7-F, let-7-R and the cDNA by denaturing at $95{ }^{\circ} \mathrm{C}$ for $3 \mathrm{~min}$, followed by 35 cycles at $95{ }^{\circ} \mathrm{C}$ for $30 \mathrm{~s}$ and $60{ }^{\circ} \mathrm{C}$ for $30 \mathrm{~s}$, and a final extension step at $60^{\circ} \mathrm{C}$ for $5 \mathrm{~min}$. PCR was performed to amplify the sequence; the PCR product was ligated into a PMD 19-T vector (TaKaRa), cloned into JM109 E. coli (Takara) and sequenced by GenScript (Nanjing, China).

\section{Prediction of target sites}

The mature let-7 miRNA sequences (UGAGGUAGUAGG UUGUAUAGU) were obtained from miRBase release 19. Two additional ECR $3^{\prime}$-UTR sequences of Amblyomma americanum, AamECR3'-UTR-1 (AF020189.1) and AamECR3'-UTR-2(AF020190.1), along with $H$. asiaticum ECR $3^{\prime}$-UTR were obtained from NCBI to predict the target sites. The miRNA target binding sites in the $3^{\prime}$-UTR of ECR were predicted using the miRNA prediction software RNAhybrid [23, 24].

\section{Dual luciferase reporter (DLR) assay}

let-7 mimic and negative control (NC) were synthesized by RiboBio (Guangzhou, China). miRNA mimics are small, chemically modified double-stranded RNAs that mimic endogenous miRNAs and enable miRNA functional analysis by upregulation of miRNA activity. The miRNA negative control is a mimic and the sequence was based on a Caenorhabditis elegans miRNA, which was not similar to mammalian or tick miRNAs. Mammalian BHK-CDC cell lines were used in the DLR assay to ensure high transfection efficiency and low background expression of let-7. ECR-A2 wild-type (WT) or mutant $3^{\prime}$-UTR was cloned into a pmirGLO vector (Promega, Madison, USA). BHK cells were transfected with $50 \mathrm{nM}$ 
miRNA mimic (final concentration) and $0.8 \mathrm{ng}$ pmirGLO reporter plasmid and mixed with $1 \mu \mathrm{l}$ of Lipofectamine ${ }^{\circledR}$ 2000 Transfection Reagent ${ }^{\circledR}$ (Invitrogen, California, USA) and $50 \mu \mathrm{l}$ of Opti-MEM Reduced Serum Medium (Gibco, California, USA) in each well of a 24-well plate. In this assay, let-7 effectively regulates the expression of the empty pmirGLO vector. Therefore, a 500-bp fragment not containing the ECR sites to which let-7 binds was cloned into the pmirGLO vector and this recombinant plasmid was designated as RP-NC. Similarly, the $3^{\prime}$-UTR of ECR was cloned into GLO plasmid named WT (3'-UTR-GLO). There were four groups in this experiment: RP-NC with miR-NC, WT with miR-NC, RP-NC with mimic, and WT with mimic. The group of $\mathrm{RP}-\mathrm{NC}$ with miR-NC was used as a blank control, and these groups of RP-NC with mimic and WT with mimic were used as the negative controls. The Dual-Luciferase ${ }^{\circledR}$ reporter assay (Promega) was performed according to the manufacturer's protocol $48 \mathrm{~h}$ after transfection. The assays were performed in triplicate for three times.

\section{Construction and transfection of plasmids}

For the construction of plasmids, a 1358-bp ECR sequence was used, including an ORF (960-bp) and 3'-UTR (398-bp), PCR was performed using cDNA and performed under the following conditions: $95{ }^{\circ} \mathrm{C}$ for 3 min followed by 35 cycles at $95{ }^{\circ} \mathrm{C}$ for $30 \mathrm{~s}, 55^{\circ} \mathrm{C}$ for $30 \mathrm{~s}$ and $72{ }^{\circ} \mathrm{C}$ for $30 \mathrm{~s}$, and a final step at $72{ }^{\circ} \mathrm{C}$ for 10 min. The sequence was cloned into PcDNA3.1 ${ }^{(+)}$plasmid (Invitrogen) using restriction sites EcoRI and NotI. The resulting plasmid was expressed in BHK-CDC cells. The PCR primers are listed in Table 1.

The cells were seeded at $10^{6}$ cells per well in 24-well plates $12 \mathrm{~h}$ before transfection. The cells were transfected with $0.8 \mu \mathrm{g}$ of plasmid and $50 \mathrm{nM}$ miRNA mimics (RIBOBIO, Guangzhou, China) per well. No-mimic treatment cells were used in the blank control. The cells were transfected with WT plasmid and mimic in the negative controls. The cells were lysed after $48 \mathrm{~h}$ in $1 \mathrm{ml}$ of Trizol. Finally, real-time PCR was performed using rat GAPDH as the reference gene.

\section{Real-time quantitative PCR}

The expression levels of let- 7 and ECR in H. asiaticum were estimated by RT-qPCR. Total RNA from different developmental stages of ticks was extracted using Trizol reagent, and the first strand cDNAs were synthesized from total RNA using stem-loop primers for let-7 or oligo dT(18) for the ECR gene. Subsequently, $1 \mu \mathrm{g}$ of total RNA was reverse transcribed using stem-loop primers (SL-primers) for generating miRNA cDNAs using PrimeScript ${ }^{\mathrm{TM}}$ RT reagent kit with gDNA Eraser (TaKaRa). The miRNA stem-loop primer and miRNA RT-qPCR primers
Table 1 Primers for this experiment

\begin{tabular}{|c|c|}
\hline Name & Sequence $\left(5^{\prime}-3\right)$ \\
\hline \multirow[t]{2}{*}{ ECR-DP } & F:CTGYGACATCGACATGTACATG \\
\hline & R:TCACTCYTGGATGTCCCARATCTC \\
\hline \multirow[t]{2}{*}{ dsECR } & $\begin{array}{l}\text { F:GGATCCTAATACGACTCACTATAGGCATCGTCTATG } \\
\text { GGTGGTGGTGTGAG }\end{array}$ \\
\hline & $\begin{array}{l}\text { R:GGATCCTAATACGACTCACTATAGGCGATGGCTGTG } \\
\text { AGGAGTGCATATTC }\end{array}$ \\
\hline \multirow[t]{2}{*}{ dsNC-1 } & F:GTAGCAGGTGTGGTTCATCC \\
\hline & R:CTGATGCATTGCCTTCGTCC \\
\hline \multirow[t]{2}{*}{ dsNC } & $\begin{array}{l}\text { F:GGATCCTAATACGACTCACTATAGGGTAGCAGGTGT } \\
\text { GGTTCATCC }\end{array}$ \\
\hline & $\begin{array}{l}\text { R:GGATCCTAATACGACTCACTATAGGCTGATGCATTG } \\
\text { CCTTCGTCC }\end{array}$ \\
\hline \multirow[t]{2}{*}{ ECR-WT-DLR } & $\begin{array}{l}\text { F:AGCTTTGTTTAAACGCCGAGATTTGGGACATCCAAG } \\
\text { AG }\end{array}$ \\
\hline & R:CTAGTCTAGACAGAAAAGAGGGTTAGATTCGC \\
\hline \multirow[t]{2}{*}{ RP-NC-DLR } & $\begin{array}{l}\text { F:AGCTTTGTTTAAACGCCGAGATTTGGGACATCCAAG } \\
\text { AG }\end{array}$ \\
\hline & R:CTAGTCTAGACAGAAAAGAGGGTTAGATTCGC \\
\hline \multirow[t]{2}{*}{$E C R-q P C R$} & F:GTGCCAGTGTAGGCGATTCAG \\
\hline & R:CTGTATGCGCTCCACCTTGC \\
\hline GSP-ECR-3UTR-F1 & F:TGGACCCGTGCAAGGTGGAG \\
\hline GSP-ECR-3UTR-F2 & F:ACAGAGCTGCGCACCTTGG \\
\hline \multirow[t]{2}{*}{$\beta$-actin } & F:CGTTCCTGGGTATGGAATCG \\
\hline & R:TCCACGTCGCACTTCATGAT \\
\hline \multirow[t]{2}{*}{ GAPDH } & F:CGTGCCGCCTGGAGAAACCTG \\
\hline & R:AGAGTGGGAGTTGCTGTTGAAGTCG \\
\hline let-7-qPCR & F:ACACTCCAGCTGGTGAGGTAGTAGGT \\
\hline miR-2a-qPCR & F:ACACTCCAGCTGGTATCACAGCCAGCTT \\
\hline miR-8-qPCR & F:ACACTCCAGCTGGTAATACTGTCAGGTA \\
\hline mir-451-qPCR & F:ACACTCCAGCTGGAAACCGTTACCATTA \\
\hline miRNA-URP & R:GTCGTATCCAGTGCAGGGTCCGAGGT \\
\hline \multirow[t]{2}{*}{ ECR-NOTI-1429 } & F:CCGGAATTCATGCGACGCAAGTGCCA \\
\hline & R:AAATATGCGGCCGCTGCATGAGCAGAAAAGAGGG \\
\hline
\end{tabular}

Note: $\mathrm{R}=\mathrm{A} / \mathrm{G}, \mathrm{W}=\mathrm{A} / \mathrm{T}, \mathrm{Y}=\mathrm{T} / \mathrm{C}$

were designed as previously described [25]. The primer sequences used in this study are listed in Table 1.

ECR primers were designed to measure ECR expression by qPCR in Table 1 . RT-qPCR for miRNA or mRNA was performed using the SYBR ${ }^{\circledR}$ Premix Ex Taq ${ }^{\mathrm{TM}}$ II Kit (TaKaRa) and an MX7500 detection system (Siskiyou, Inc., Grants Pass, OR, USA).

\section{Injection of miRNA}

To further validate the role of let-7, miRNA was overexpressed using let-7 agomir in vivo. agomir is a chemicallymodified double-strand miRNA mimic. Its antisense strand is modified: 2 phosphorothioates at the $5^{\prime}$ end, 4 phosphorothioates at the $3^{\prime}$ end, $3^{\prime}$ end cholesterol group and full length nucleotide $2^{\prime}$-methoxy modification. The 
developmental period from fully-engorged larvae to unfed nymphs was 7-10 days and from fully-engorged nymphs to unfed adults was 30-45 days. The nymphs received the miRNAs 10 days after blood-feeding and were reared in the laboratory.

The miRNA or miRNA negative control (miRNANC) (RiboBio) was microinjected into the haemocoel of engorged nymphs at a dose of $400 \mathrm{~nm}$ in $0.5 \mu \mathrm{l}$ [26]. Each miRNA was injected into 100 nymphs 10 days after blood-feeding. Control ticks were injected with an equal volume of PBS buffer (pH 7.4). In this experiment, the ticks were divided into four groups as follows: non-injection (untreated), PBS-injection (PBS), NCmiRNA-injection (miR-NC) and let-7-agomir-injection (agomir). Twenty-four hours after injection, dead ticks were removed and live ticks were maintained at a temperature of $25{ }^{\circ} \mathrm{C}$ and relative humidity of $75-85 \%$. The total RNA from three live ticks was extracted for RTqPCR. Three miRNAs (miR-2a, miR-8 and miR-451) were tested by RT-qPCR. The miR-2a loop primer (5'GTC GTA TCC AGT GCA GGG TCC GAG GTA TTC GCA CTG GAT ACG AC GCT CAT CA-3'), the miR-8 loop primer (5'-GTC GTA TCC AGT GCA GGG TCC GAG GTA TTC GCA CTG GAT ACG AC GAC ATC TT-3') and the miR-451 loop primer (5'-GTC GTA TCC AGT GCA GGG TCC GAG GTA TTC GCA CTG GA TAC GAC AAA CTC AG-3') for miR-2a, miR-8 and miR-451 first-strand cDNA, respectively. Ticks were observed on a daily basis and survived for 1 month. Dead ticks were removed and the survival rates were calculated after 1 month.

Antagomirs also known as anti-miRs or blockmirs are a class of chemically engineered oligonucleotides that prevent other molecules from binding to a desired site on an mRNA molecule. Antagomirs are used to silence endogenous microRNA (miR) [27]. The miRNA antagomir or miRNA negative control (miRNA-NC) (RiboBio) was micro-injected into the haemocoel of engorged nymphs at a dose of $400 \mathrm{~nm}$ in $0.5 \mu \mathrm{l}$. Each miRNA was injected into 100 nymphs 10 days after blood-feeding. Control ticks were injected with an equal volume of PBS buffer ( $\mathrm{pH}$ 7.4). In this experiment, the ticks were divided into four groups as follows: non-injection (untreated), PBSinjection (PBS), NC-miRNA-injection (miR-NC) and let-7-antagomir-injection (ant-let-7). Twenty-four hours after injection, dead ticks were removed and live ticks were maintained at a temperature of $25{ }^{\circ} \mathrm{C}$ and relative humidity of $75-85 \%$.

\section{RNA interference}

A 572-bp fragment corresponding to the core region of ECR and a 539-bp NC fragment were amplified by
PCR. NC is a plant-derived gene. The primers used were ECR-RNAi-F, ECR-RNAi-R for synthetic dsRNA of ECR named dsECR, and NC-RNAi-F, NC-RNAi-R for dsRNA of NC named dsNC. PCR was performed under the following conditions: $95^{\circ} \mathrm{C}$ for 3 min followed by 35 cycles at $95{ }^{\circ} \mathrm{C}$ for $30 \mathrm{~s}, 55^{\circ} \mathrm{C}$ for $30 \mathrm{~s}$ and $72{ }^{\circ} \mathrm{C}$ for $30 \mathrm{~s}$, with a final step at $72{ }^{\circ} \mathrm{C}$ for $10 \mathrm{~min}$. The dsRNA was prepared using the T7 RiboMax ${ }^{\mathrm{TM}}$ express RNAi system (Promega) according to the manufacturer's instructions. In this experiment, the ticks were divided into four groups as follows: non-injection (untreated), PBS-injection (PBS), dsNC-injection (dsNC) and dsECR-injection (dsECR). Synthetic dsRNAs were microinjected into 100 nymphs. The nymphs received the dsRNA 10 days after bloodfeeding and were reared in the laboratory. A total of $2 \mu \mathrm{g}$ of dsRNA in $0.5 \mu \mathrm{l}$ of water was injected into each tick and the negative control groups included a PBS-injection group (untreated), a group receiving PBS (PBS group), and a group receiving NC dsRNA (ds-NC group). Subsequently, the expression of ECR genes was measured by quantitative real-time PCR. Statistical analysis was conducted to assess the effect of RNA silencing on molting.

\section{Hormone treatment}

Ten days after blood-feeding, the nymphs were injected with either 3 ug of $20 \mathrm{E}$ dissolved in $0.5 \mathrm{ul}$ of water (experimental group) or water of DMSO (control group). let-7 and ECR were tested by qPCR at 24 and $48 \mathrm{~h}$. $\beta$-actin was amplified as internal controls. The relative expression of miRNAs and ECR was calculated by the $2^{-\Delta \Delta C t}$ method.

\section{Statistical analysis}

All data sets are shown as means $\pm \operatorname{SEM}(n \geq 3)$. The dual luciferase reporter (DLR) assay and quantitative real-time PCR results were analyzed using a two-tailed unpaired Student's t-test as detailed in the figure legends by GraphPad Prism 6 software (GraphPad Software, San Diego, CA, USA). Significance was set at $P<0.05$.

\section{Results}

\section{Analysis of target genes and let-7 binding sites}

The partial sequence of ECR include a 960-bp opening reading frame (ORF) and a 398-bp 3'-UTR containing a poly (A) terminal sequence (HaaECR, GenBank: MF135614.2). Additionally, we identified three AamECR isoforms (AamECRA1, AamECRA2 and AamECRA3) and the AamECR3'-UTR isoforms in $H$. asiaticum. The structure of the gene encoding HaaECR is shown in Fig. 1, redrawn from the study by Guo et al. [28]. AamECR and $H a a \mathrm{ECR}$ were used for predicting the let-7 binding site and these sequences contained the same let-7 binding site (Fig. 1). 


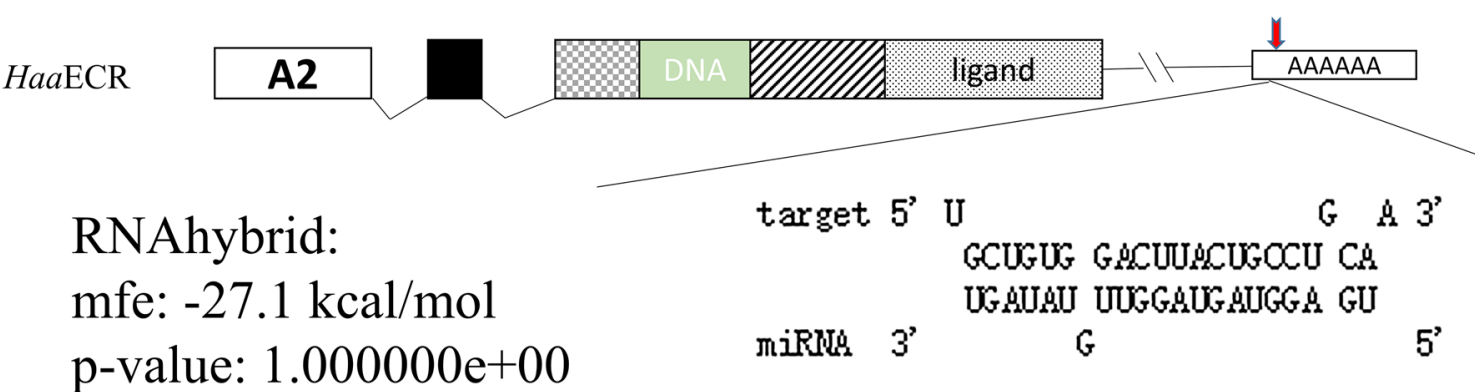

Fig. 1 Predicted let-7 target sites in the ECR gene by RNAhybrid. The red triangle indicates the binding sites for let-7 in 3'-UTR. HaaEcR was identified to predict the binding site with let-7 in this experiment
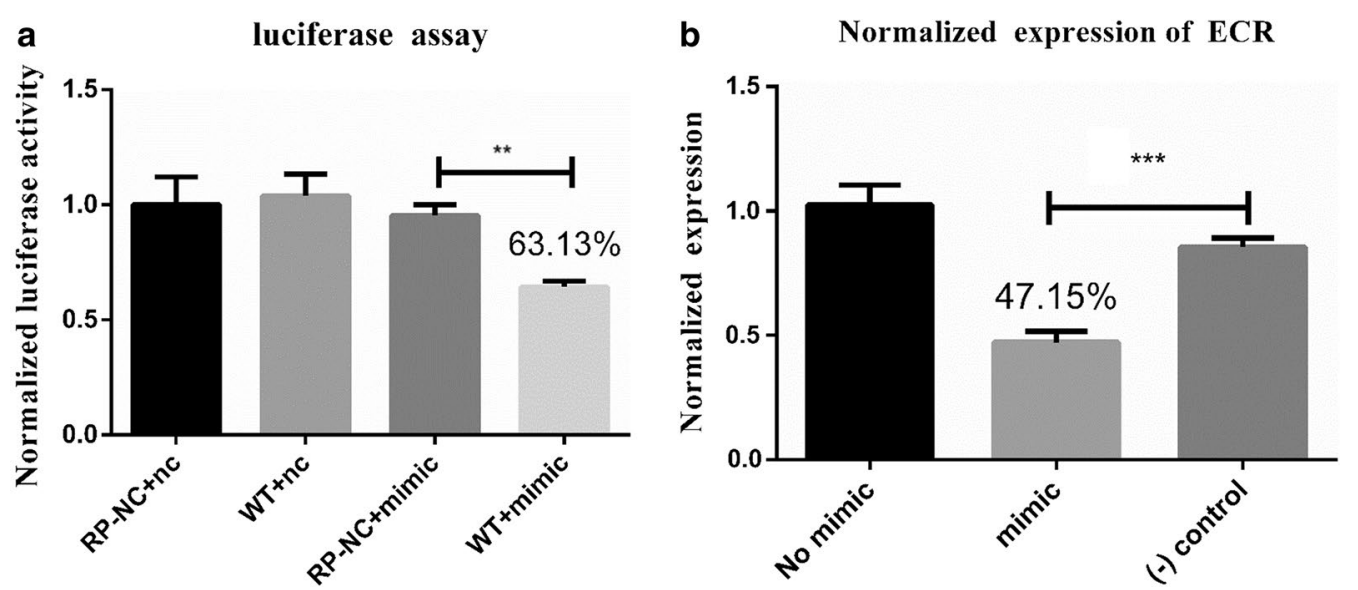

Fig. 2 a ECR is a target of let-7. Dual luciferase reporter assay results are represented as mean \pm SEM of triplicate samples. $\mathbf{b}$ The recombinant plasmid pcDNA3.1 containing the ECR gene, including a 960-bp ORF and a 298-bp 3-UTR, was transfected into BHK cells. The expression of ECR was measured by $\mathrm{qPCR}$. Data are presented as the mean \pm SEM of triplicate samples. ${ }^{*} P<0.05,{ }^{* *} P<0.01,{ }^{* * *} P<0.001$ (Student's t-test)

\section{MiRNA represses ECR expression in BHK-CDC cells}

The DLR assay was used to assess the effect of let-7 on ECR expression in BHK-CDC cells. The plasmids with mimic or NC sequences were transfected into BHK-CDC cells. Compared with the RP-NC+NC group, the ratio of luciferase activity was 63\% (Fig. 2a). The results showed that the ratio of luciferase activity in the $\mathrm{WT}+$ mimic group was different from that in the other groups.

To determine whether or not let-7 could repress ECR expression, the eukaryotic expression vector containing the 1358-bp ECR gene, including a 960-bp CDS and a 398-bp 3'-UTR, was transfected with the let-7 mimic into BHK-CDC cells (Fig. 2b). ECR expression in the treatment group was $47 \%$ when compared with the control group. These results demonstrated that ECR was the target of let-7 and that let-7 repressed ECR expression in BHK cells.

\section{Analysis of the expression of let-7 and ECR}

Total RNA was extracted from different stages of $H$. asiaticum and the expression levels of let-7 and ECR were amplified by RT-qPCR at two molting stages: from larvae to nymphs and from nymphs to adults. $\beta$-actin was amplified as internal controls. Relative expression of miRNAs and ECR was calculated by the $2^{-\Delta \mathrm{Ct}}$ method. In Fig. $3, \mathrm{~L}-1$ and L-2 correspond to engorged larvae before and after molting, respectively, and N-1 and N-2 correspond to engorged nymphs before and after molting, respectively. For both engorged larvae and engorged nymphs, the levels of ECR expression before molting were higher than those after molting $\left(t_{(4)}=6.920, \quad P=0.0023 ; t_{(6)}=8.520, \quad P<0.001\right)$, whereas the expression levels of let- 7 before molting were lower than after molting $\left(t_{(6)}=8.520, P<0.001 ; t_{(6)}=3.860\right.$, $P<0.0084)$. The differences were significant. Moreover, a correlation in expression levels between let-7 and ECR and a different changing tendency of the expression level were observed. Therefore, this analysis suggests that there is an interaction between let-7 and ECR, and this interaction affects molting. Such a difference was not due to the digestion of the tick blood meal before and after molting because the levels of let-7 and HaaECR were low in the host blood. 


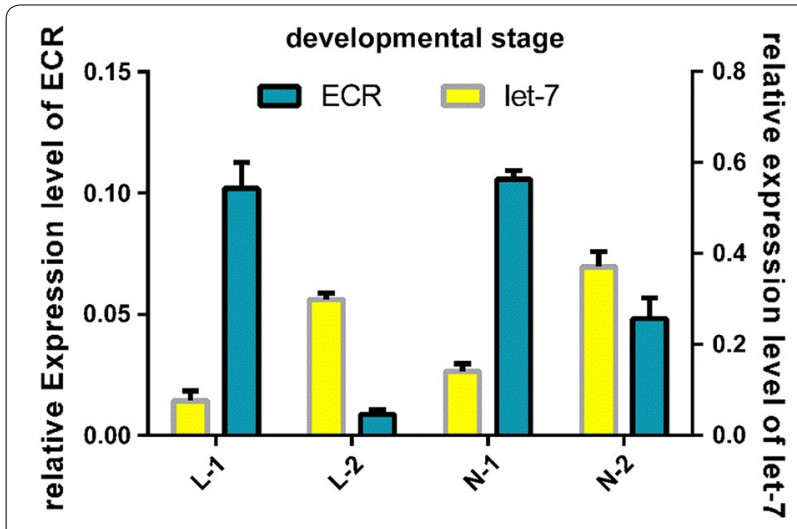

Fig. 3 Analysis of the expression of let-7 and ECR. L-1 and L-2 correspond to engorged larvae before and after molting, respectively. $\mathrm{N}-1$ and $\mathrm{N}-2$ correspond to engorged nymphs before and after molting, respectively. Data are presented as the mean \pm SEM of triplicate samples

\section{Overexpression of let-7 in $\mathrm{H}$. asiaticum}

let-7 agomir was used to elucidate the biological function of let-7 in ticks. After $24 \mathrm{~h}$ of miRNA injection, the expression of let-7 in the agomir group was increased approximately 6935-fold and the expression of ECR was decreased approximately 6.04-fold compared to the miR-NC group. Three miRNAs (miR-2a, miR- 8 and miR451) were tested by RT-qPCR, and the expression levels of mir- $2 \mathrm{a}$ and mir- 8 were downregulated by approximately 74 and 52\%, respectively, in the agomir group compared to the untreated group, whereas the expression of miR-451 was not significantly changed (Fig. 4a). There were significant differences in the expression levels of ECR between the agomir group and the other groups $\left(t_{(6)}=15.56, P<0.001\right.$ ) (Fig. $\left.4 \mathrm{~b}\right)$. The tick survival rate in the let-7 overexpression group was approximately $30 \%$ less in comparison to the miR-NC group. However, the survival rate in the untreated group was greater than those in other groups (Fig. 4c). There were significant differences between the agomir group and the other groups $\left(t_{(4)}=3.514, P<0.0024\right)$, showing that injection caused physical injury to ticks.

Moreover, our results showed that the period of molting was also affected by agomir treatment. In engorged ticks, the average number of days of molting was

\section{a \\ Normalized expression of miRNAs}
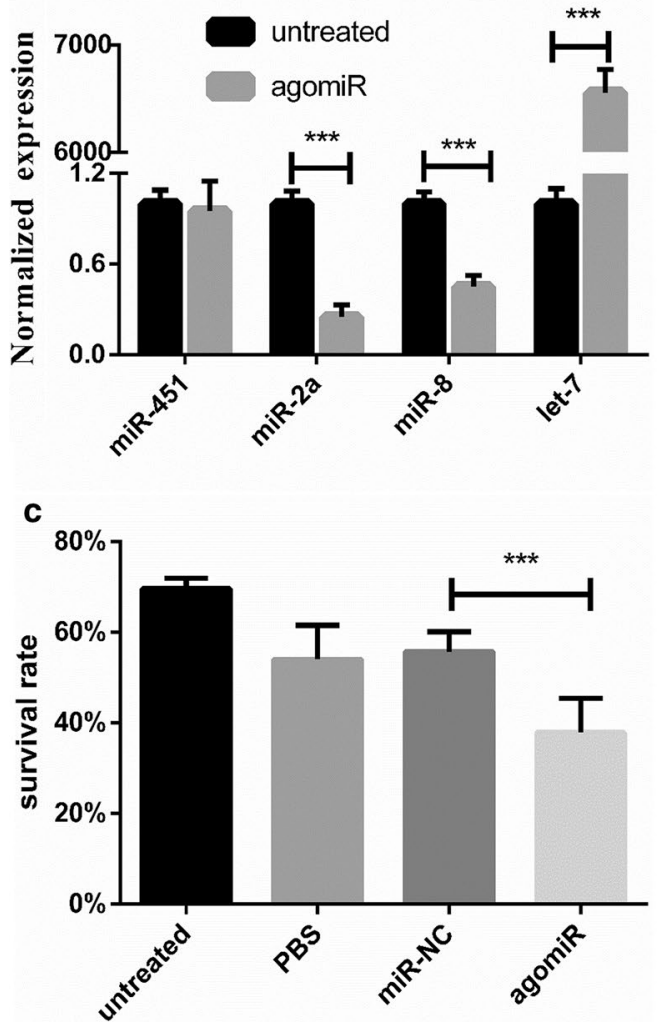
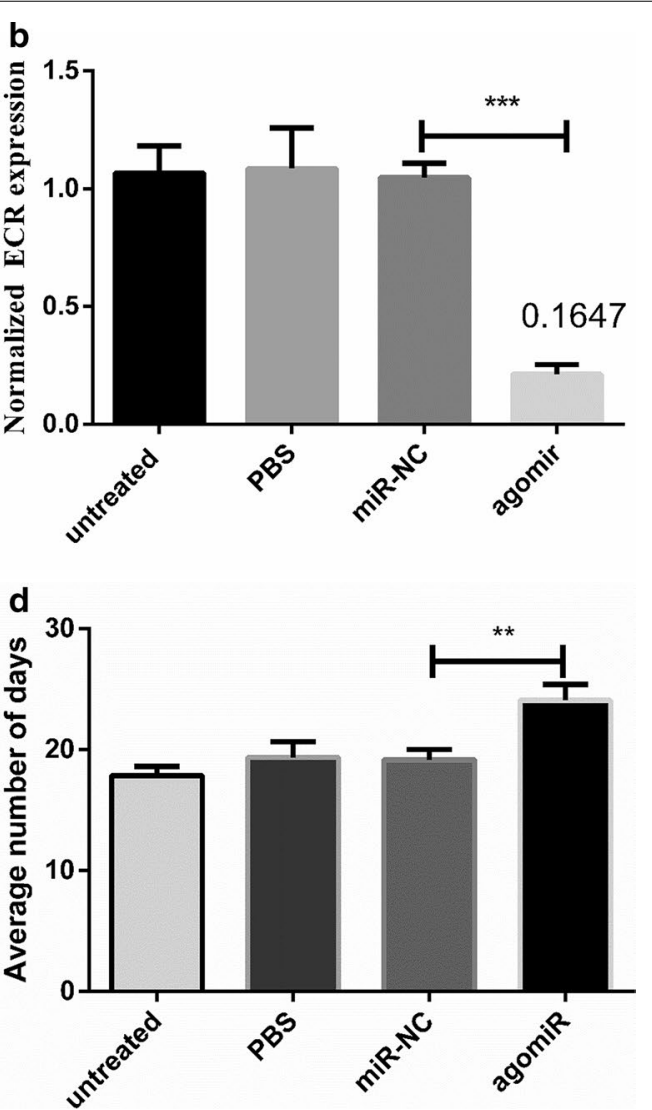

Fig. 4 a The miRNAs were tested in the agomir and untreated groups. b The expression of ECR was tested $24 \mathrm{~h}$ after injection of agomir. $\mathbf{c}$ Effect of let-7 overexpression on survival rate. $\mathbf{d}$ Average number of days of molting after injection with agomir. Data are presented as the mean $\pm S E M$ of triplicate samples. ${ }^{*} P<0.05,{ }^{* *} P<0.01,{ }^{* * *} P<0.001$ 


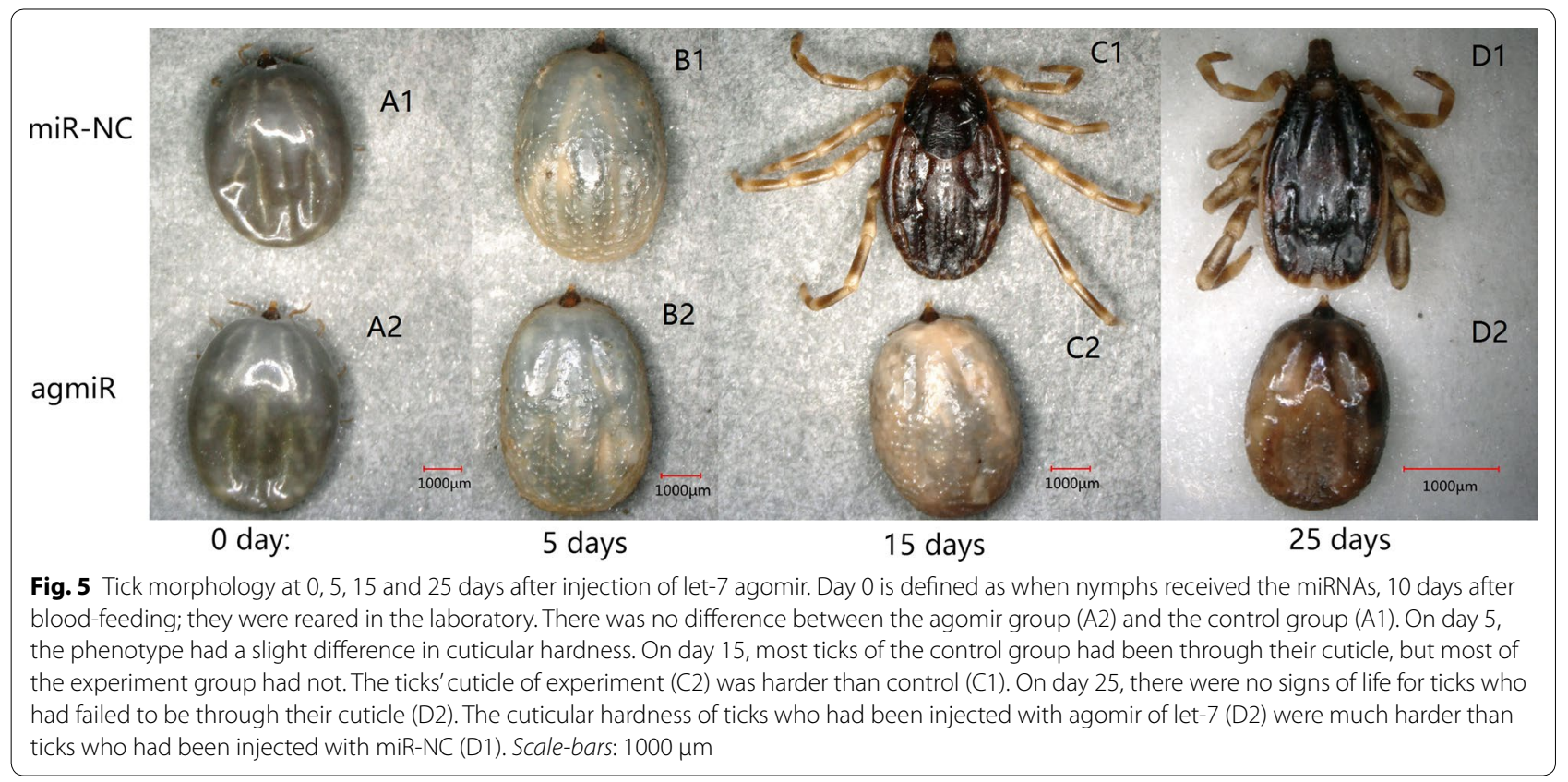

Table 2 The phenotype of ticks in the overexpression experiment

\begin{tabular}{|c|c|c|c|c|}
\hline & \multicolumn{4}{|l|}{ Group } \\
\hline & Untreated & PBS & miR-NC & agomir \\
\hline Average number of days & $18.20 \pm 1.02^{\mathrm{a}}$ & $19.37 \pm 1.05^{\mathrm{a}}$ & $19.16 \pm 0.71^{\mathrm{a}}$ & $24.43 \pm 0.62^{b}$ \\
\hline Survival rate (\%) & $67.385 \pm 1.085^{a}$ & $54.914 \pm 0.184^{b}$ & $56.138 \pm 0.736^{b}$ & $38.469 \pm 1.182^{\circ}$ \\
\hline
\end{tabular}

Notes: Each data point is the mean \pm SEM of three independent experiments $(n=3)$. Different letters indicate significant differences on the same line $(P<0.05)$

$18.20 \pm 1.02$ days in the untreated group, $19.37 \pm 1.05$ days in the PBS group, $19.16 \pm 0.71$ days in the miR-NC group, and $24.43 \pm 0.62$ days in the agomir group. The periods of molting in the PBS and miR-NC groups were not significantly different from that in the untreated group $(P>0.05)$, indicating that the period from engorgement to molting was not affected by the injection operation nor non-specific miRNA injection (Fig. 4d). However, molting was significantly delayed by the treatment of agomir $\left(t_{(4)}=5.874, P=0.0042\right)$, demonstrating that let-7 overexpression prolonged the molting period in ticks.

Tick morphology was evaluated at 0, 5, 15 and 25 days post-injection (pi) of let-7 agomir or miR-NC (Fig. 5). Tick phenotype did not change until day 5 pi but presented significant difference after 5 days pi. Ticks from the agomir group exhibited a conspicuous "molting defect" phenotype, in which the old cuticle was not shed. These results demonstrate that the overexpression of let- 7 could reduce the survival rate and increase the molting period. The results are shown in Table 2.

\section{ECR interference in H. asiaticum}

The biological function of ECR was confirmed in vivo by injecting dsECR in engorged nymphs to silence the ECR gene. After the treatment, the expression levels of ECR and let-7 were measured by real-time PCR $24 \mathrm{~h}$ post-injection. ECR expression in the dsECR group was decreased approximately by $42 \%$ and let- 7 was upregulated approximately by $37 \%$ compared to the untreated group (Fig. 6a). The ratio of let-7 to ECR was increased $\left.t_{(4)}=5.492, P=0.017\right)$ approximately 1.37 times in the dsECR group compared with the untreated group (Fig. 6b). The effect of RNA interference on the survival rate is shown in Fig. 6c. The survival rate in the dsECR group was significantly decreased by approximately $8 \%$ compared with the dsNC group $\left(t_{(4)}=5.192\right.$, $P=0.0067)$. In engorged ticks, the average number of days of molting was $20.34 \pm 1.46$ days in the untreated group, $18.48 \pm 2.08$ days in the PBS group, $21.49 \pm 0.42$ days in the miR-NC group and $23.92 \pm 0.74$ days in the agomir group (Fig. $6 \mathrm{~d}$ ). The results are shown in Table 3. 

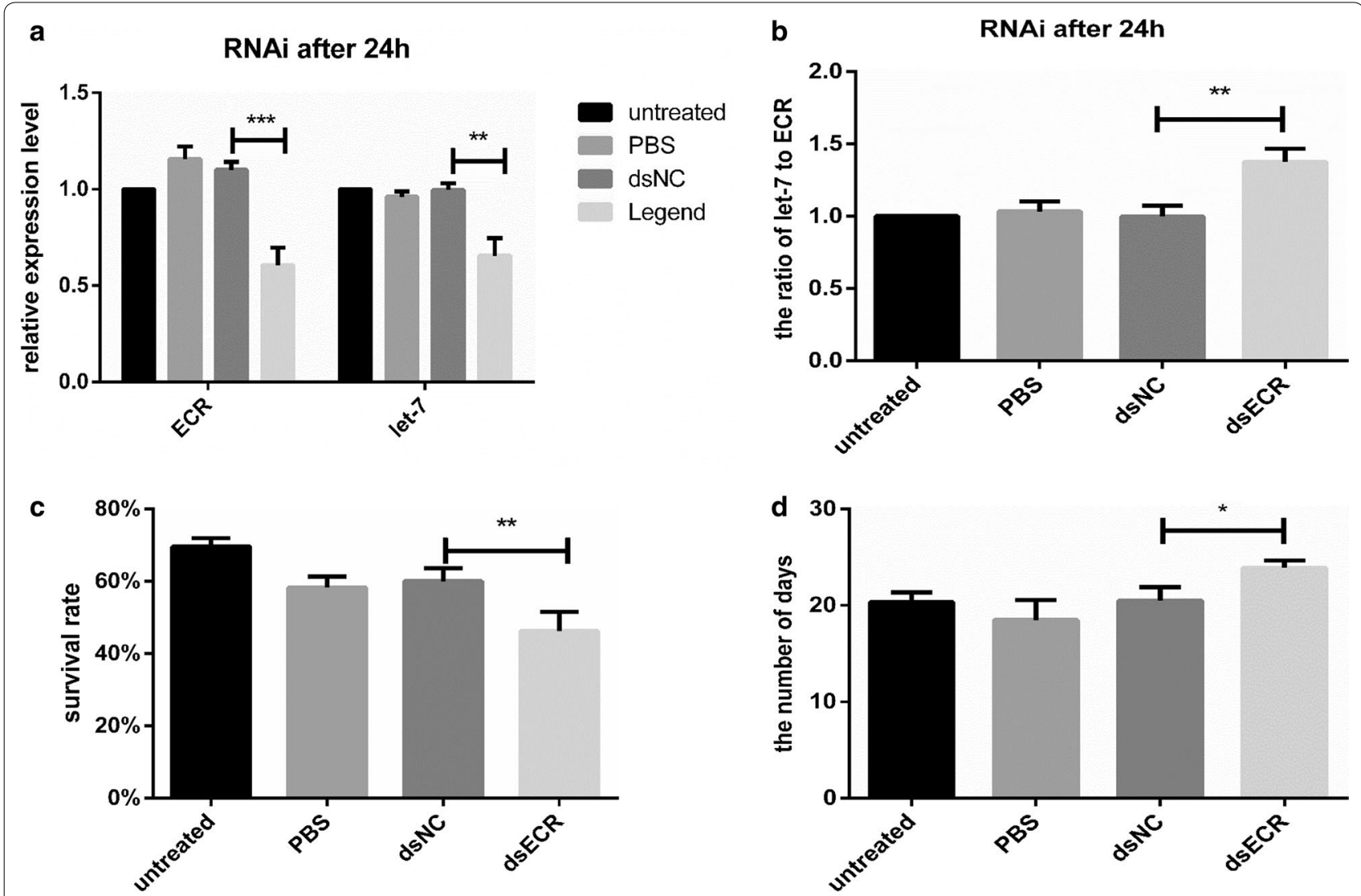

Fig. 6 a ECR expression was determined $24 \mathrm{~h}$ after injection of dsRNA. $\mathbf{b}$ The ratio of let-7 to ECR $24 \mathrm{~h}$ after injection of dsRNA. Effect of RNA interference on survival rate. $\mathbf{d}$ Average number of days of molting after injection of dsRNA. Data are presented as the mean \pm SEM of triplicate samples. ${ }^{*} P<0.05,{ }^{* *} P<0.01,{ }^{* * *} P<0.001$

Table 3 The phenotype of ticks in the RNAi experiment

\begin{tabular}{|c|c|c|c|c|}
\hline & \multicolumn{4}{|l|}{ Group } \\
\hline & Untreated & PBS & $\mathrm{dsNC}$ & dsECR \\
\hline Average number of days & $20.34 \pm 1.46^{\mathrm{a}}$ & $18.48 \pm 2.08^{\mathrm{a}}$ & $21.49 \pm 0.42^{\mathrm{a}}$ & $23.92 \pm 0.74^{b}$ \\
\hline Survival rate $(\%)$ & $62.468 \pm 3.146^{\mathrm{a}}$ & $50.451 \pm 0.791^{\mathrm{a}}$ & $54.264 \pm 1.304^{\mathrm{a}}$ & $45.614 \pm 3.079^{b}$ \\
\hline
\end{tabular}

Notes: Each data point is the mean \pm SEM of three independent experiments $(n=3)$. Different letters indicate significant differences on the same line $(P<0.05)$

Let-7 silence by antagomir

To explore the effect of let-7 silencing in ticks, a let-7 antagomir (ant-let-7) was used in this experiment to knock down the expression of let-7. The missense sequence of let- 7 was used as the negative control of the antagomir. Compared with $\mathrm{NC}$, the let-7 expression of antagomir injection group was knocked down approximately to $59 \%\left(t_{(4)}=6.961, P=0.022\right)$ (Fig. $7 \mathrm{a}$ ) at $24 \mathrm{~h}$ and haaECR increased 1.26 times $\left(t_{(4)}=4.908, P=0.0080\right.$ ) (Fig. $7 \mathrm{~b}$ ). The effect of let- 7 interference on the survival rate is shown in Fig. 7c. The survival rate in the ant-let-7 group was not significantly different from the $\mathrm{NC}$ group $\left(t_{(4)}=1.278, P=0.2702\right)$. Moreover, our results showed that the period of molting was also affected by antagomir treatment. In engorged ticks, the average number of days of molting was $18.44 \pm 0.4075(n=3)$ days in the untreated group, $20.32 \pm 0.7421(n=3)$ in the miR-NC group and $16.04 \pm 0.5410(n=3)$ days in the antagomir group (Fig. $7 d$ ). The results are shown in Table 4.

\section{E influences expression of let-7 and ECR}

Compared with the DMSO-injected group, expression of let-7 was downregulated by approximately 48 and $30 \%$ at 24 and $48 \mathrm{~h}$, respectively; ECR was upregulated 

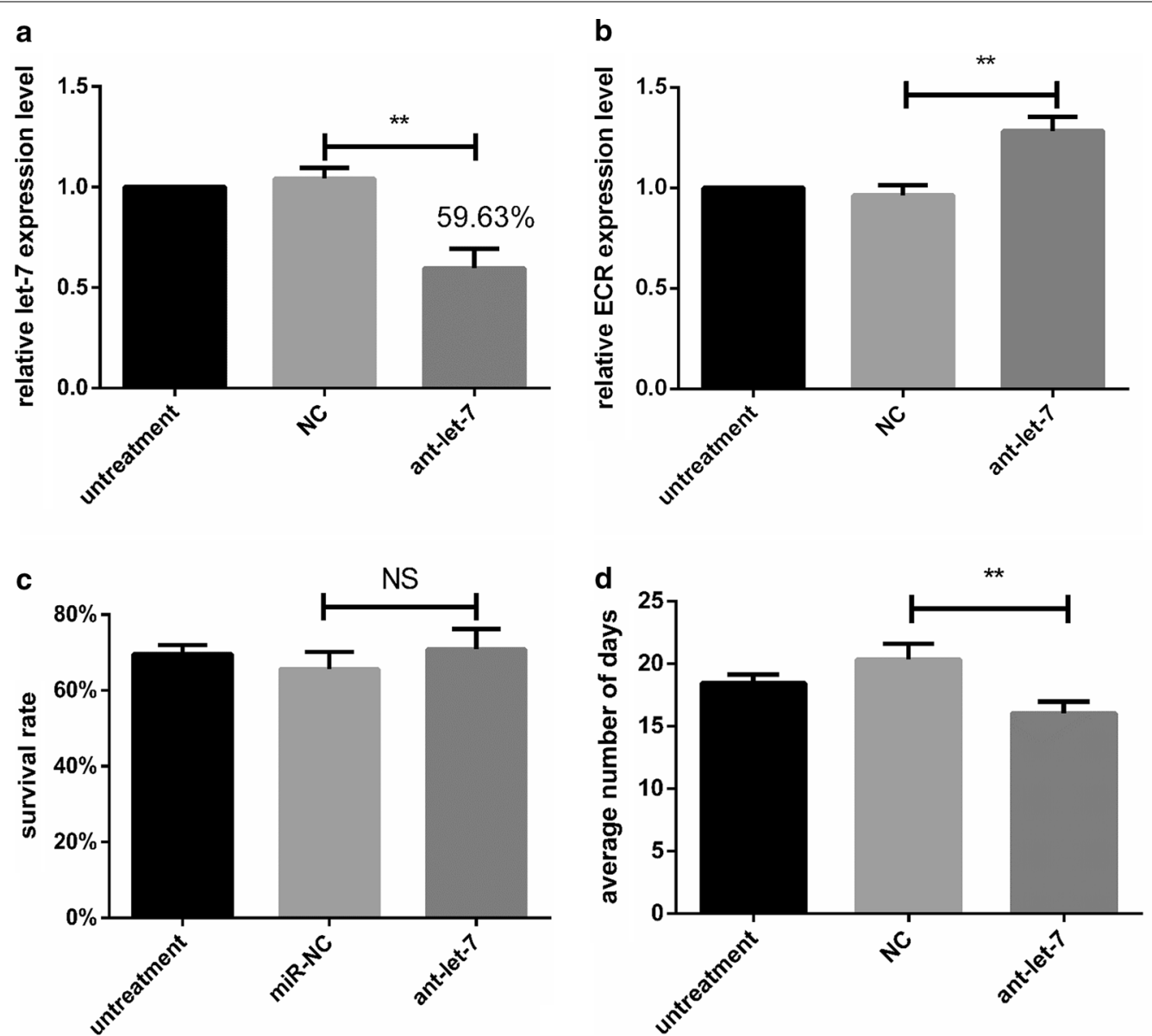

Fig. 7 a ECR expression was determined $24 \mathrm{~h}$ after injection of antagomir. b Let-7 expression was evaluated $24 \mathrm{~h}$ after injection of antagomir. c Effect of miRNA interference on survival rate. $\mathbf{d}$ Average number of days of molting after injection of antagomir. Data are presented as the mean \pm SEM of triplicate samples. ${ }^{*} P<0.05,{ }^{*} P<0.01$, NS, not significant

Table 4 The phenotype of ticks in the let-7 disturbed experiment

\begin{tabular}{llll}
\hline & Group & & \\
\cline { 2 - 4 } & Untreated & miR-NC & ant-17 \\
\hline $\begin{array}{l}\text { Average number of } \\
\text { days }\end{array}$ & $18.44 \pm 0.4075^{\mathrm{a}}$ & $20.32 \pm 0.7421^{\mathrm{a}}$ & $16.04 \pm 0.5410^{\mathrm{b}}$ \\
\begin{tabular}{l} 
Survival rate (\%) \\
\hline
\end{tabular} & $69.672 \pm 1.335^{\mathrm{a}}$ & $65.487 \pm 2.603^{\mathrm{a}}$ & $70.864 \pm 3.074^{\mathrm{a}}$ \\
\hline
\end{tabular}

Notes: Each data point is the mean \pm SEM of three independent experiments $(n=3)$. Different letters indicate significant differences on the same line $(P<0.05)$

by approximately 21 and $16 \%$ at 24 and 48 h, respectively (Fig. 8a, b).

\section{These miRNAs were analyzed by $\mathbf{3}^{\prime}$-UTR of ECR}

All miRNAs from Ixodes scapularis in miRBase web and 3 '-UTR of ECR were analyzed by RNAhybrid software (Fig. 9a). Then, the expression of these miRNAs was tested by qPCR and it was found that five miRNAs
(miR-2a, miR-2b, miR-10, miR-305 and miR-5312) were upregulated when let- 7 was silenced; these were downregulated when let-7 was overexpressed (Table 5). RNAhybrid software Luciferase assays showed that these five miRNAs (miR-2a, miR-2b, miR-10, miR-305 and miR-5312) can regulate the expression of ECR in vivo (Fig. 9b).

\section{Discussion}

The steroid hormone 20E triggers significant developmental changes in Drosophila, including molting and metamorphosis, and provides a model system for defining the developmental and molecular mechanisms of steroid signaling. 20E acts via a heterodimer of two nuclear receptors, the ecdysone receptor (ECR) and ultraspiracle, and directly regulates target gene transcription [29-31].

ECR is a conserved gene in arthropods, and either two or three isoforms are present. In D. melanogaster, three 

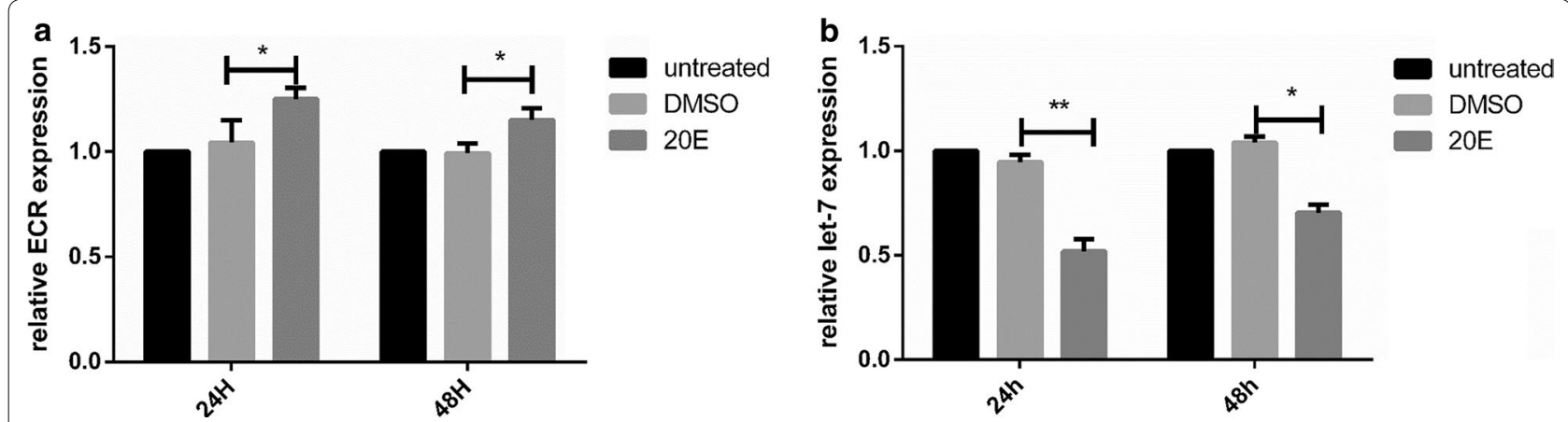

Fig. 8 a ECR expression was determined $24 \mathrm{~h}$ after injection of 20E. b Let-7 expression was evaluated $24 \mathrm{~h}$ after injection of 20E. Data are presented as the mean \pm SEM of triplicate samples. ${ }^{*} P<0.05,{ }^{* *} P<0.01$

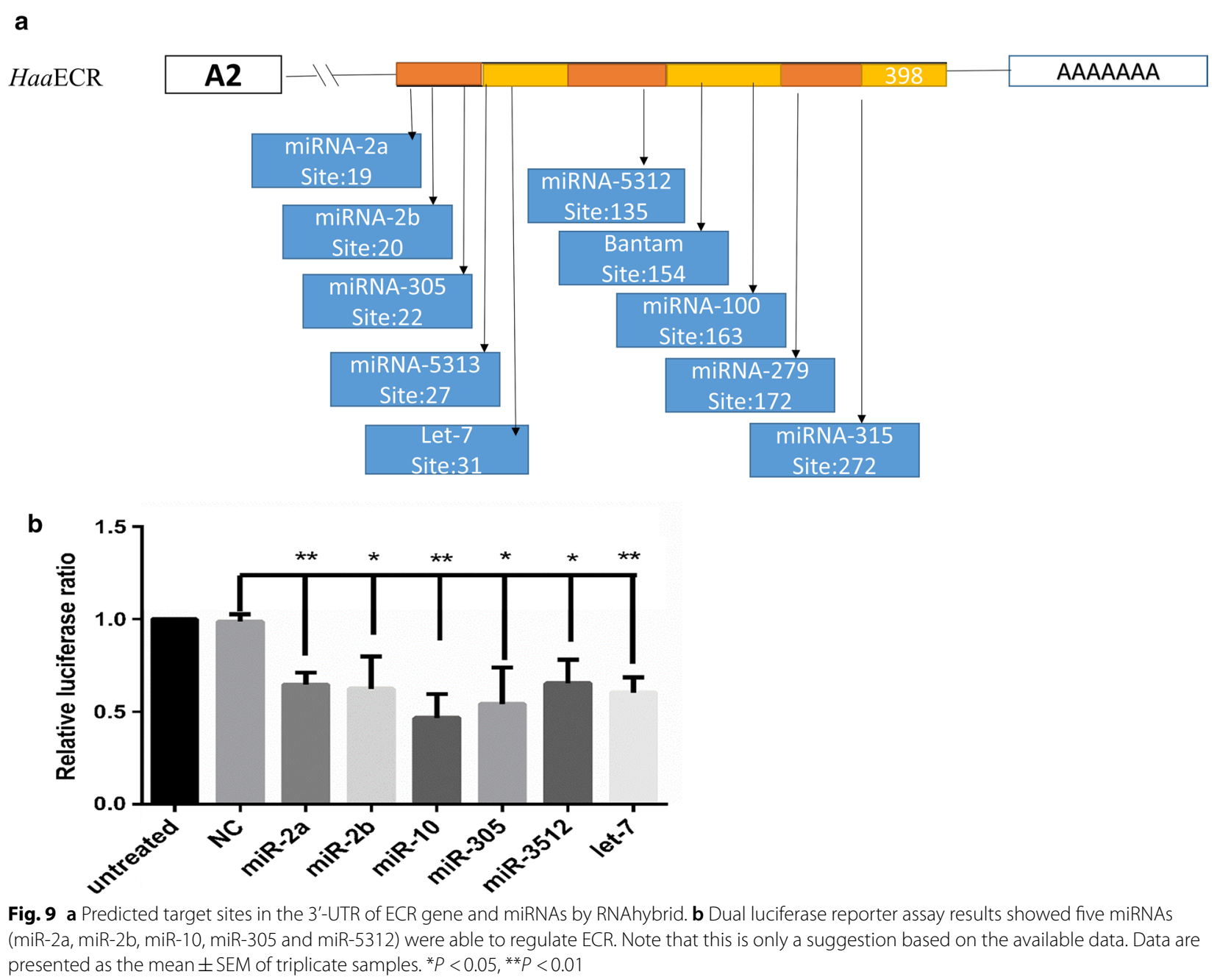

nuclear hormone receptor isoforms, ECR-A, ECR-B1and ECR-B2, are encoded by the ECR gene [30, 32]. In Ixodida, AamECR cDNA encodes three isoforms [28]. AamECRA1 and AamECRA3 have a unique 5'-sequence and contain a 3218-bp 3-UTR, which diverged to produce a unique 1166-bp terminus, 3-UTR-1, or a unique 618-bp terminus, $3^{\prime}$-UTR-2. However, there is evidence that AamECR2 has a poly (A) sequence in the $3^{\prime}$-UTR 
Table 5 miRNA relative expression level

\begin{tabular}{lll}
\hline & Agomir & Antagomir \\
\hline let-7 & Up & Down \\
miR-2a & Down & Up \\
miR-2b & Down & Up \\
miR-10 & Down & Up \\
miR-305 & Down & Up \\
miR-5313 & Up & Up \\
miR-5312 & Down & Up \\
miR-279 & Down & Down \\
miR-100 & Up & Up \\
bantam & Down & Down \\
miR-315 & Down & ns \\
\hline
\end{tabular}

Notes: miRNAs were tested when let-7 is overexpressed and knocked down. Compared with the group of injected miRNA-NC, "down" means that the expression of miRNAs is downregulated, "up" means that the expression of miRNAs is upregulated and "ns" means not significant

region. A previous study indicated that a partial 1429-bp ECR sequence contained poly (A) in the $3^{\prime}$-UTR region, including a 960-bp partial open reading frame (ORF) and 398-bp $3^{\prime}$-UTR. In addition, the $3^{\prime}$-UTR sequence of AamECR2 was different from the $3^{\prime}$-UTR-1 and 3'-UTR-3 of AamECR. The degree of homology between the amino acid sequences of HaaECR (ATA58050.1) and AamECR (AAB94567.1, AAB94566.1) was approximately 95\% using Blast from NCBI. Therefore, the partial ECR sequence ECR sequence corresponding to ECR-A2 may have a biological function in $H$. asiaticum.

Previous studies have reported that many microRNAs could bind to sites located in the $3^{\prime}$-UTR region of target genes but only a few miRNAs could bind to the $5^{\prime}$-UTR or CDS of target genes [33-35]. miRNA regulates gene expression at the post-transcriptional and translational levels [36, 37]. A previous study, conducted using Bombyx mori, demonstrated that let-7 plays a fundamental role in molting in arthropods [16]. Our results indicate that let-7 sequences are conserved among ticks. For this reason, the complete $A a m E C R$ gene sequence and the partial HaaECR-A2 gene sequence were selected for analysis of the binding sites using RNAhybrid software, and our results indicated that these sequences have the same binding sites in the $3^{\prime}$-UTR, suggesting that the let-7 binding sites in HaaECR are located in the $3^{\prime}$-UTR. For this reason, the $3^{\prime}$-UTR of HaaECR was used in the dual luciferase reporter assay. However, because the bioinformatics analysis indicated that the multiple cloning site (MCS) of the pmirGLO reporter vector interacted with let-7, the sequence that did not interact with let-7 was cloned into the pmirGLO reporter vector to create the recombinant plasmid designated RP-NC. The result of the dual luciferase reporter assay showed that the let-7 binding sites are located in the $3^{\prime}$-UTR (Fig. 2a). The PcDNA3.1 expression vector of ECR along with the let-7 mimic was co-transfected into BHK-CDC cells to confirm the hypothesis that let-7 regulates the expression of ECR in eukaryotic cells (Fig. 2b). The results of the reporter assay using a eukaryotic expression system demonstrated that HaaECR was the target of let-7 and the binding sites are located in the $3^{\prime}$-UTR of HaaECR.

Previous studies reported that the expression of miRNAs involved in the ecdysone pathway, including let7, mir-100 and mir-125, are upregulated by the binding of Broad-Complex with 20E in Drosophila [21,36]. The expression level of let-7 was higher before molting than after molting for both larvae and nymphs whereas ECR expression was lower before molting than after molting.

There is strong evidence that $20 \mathrm{E}$, as observed for other steroid hormones, regulates molting in ticks via a negative feedback mechanism. In normal physiological conditions, 20E binds to the heterodimer ECR-USP/RXR (ultraspiracle/retinoid $\mathrm{X}$ receptor) to produce ECR, E75 and Broad-Complex $\mathrm{C}$ (BR-C), and so on in the normal range. The signal casing by heterodimer is transmitted to the nucleus, activates the expression of downstream genes, and causes the ecdysis cascade reaction in the $20 \mathrm{E}$ pathway [38, 39]. However, the overexpression of let-7 causes the downregulation of ECR and affects the signal transduction pathway of ECR. For this reason, the overexpression of let-7 can downregulate the ECR gene, affecting its signal transduction pathway, and ultimately affects mortality and the period of molting in ticks. However, among the miRNAs tested in this study, miR-2a and mir-8 were downregulated and mir-451expression was not changed. A recent study reported that a target gene of miR-2a was membrane-bound trehalase and a target gene of miR-8 was phosphoacetylglucosamine. In addition, these miRNAs and their respective targets belong to the chitin biosynthesis pathway and respond to $20 \mathrm{E}$ signaling [40]. However, there were no studies showing that miR-451 belongs to the molting pathway and related pathways. Therefore, agomir, which increases the expression of let-7, only affects other miRNAs which is along with let-7 in one signaling pathway and does not change the miRNAs expression of other pathways.

The effect of RNA interference on the expression of let-7 and ECR was also evaluated. Both let-7 and ECR were downregulated by dsECR injection. However, the ratio of let-7 to ECR was increased $(P<0.05)$ approximately 1.37 times in the dsECR group compared with the untreated group. These results can explain the fact that ECR is regulated by let-7. In addition, phenotypic changes indicated that ECR plays a significant role in the process of molting of ticks (Fig. 6). 
In this experiment, antagomir was used to knockdown let-7 expression. In spite of the fact that let-7 was downregulated and ECR was upregulated, the rate of survival was not different between the ant-let- 7 group and miR-NC group (Fig. 7d). However, RNAhybrid software and Luciferase assays revealed that these five miRNAs (miR-2a, miR-2b, miR-10, miR-305, and miR-5312) can regulate the expression of ECR in vivo (Fig. 9). Recently, research in human diseases and cancer has proved that miRNAs-miRNAs can build a synergistic network via co-regulation function modules [41-43]. However, in the life-cycle of ticks, we can find molting plays a significant role in molting. As a reporter protein of $20 \mathrm{E}$ in the process of molting, ECR may be regulated by many co-regulatory factors. The limited miRNAs are thought to be able to control the larger set of genes through synergism, in which multiple miRNAs work synergistically to control individual genes modules. These five miRNAs may be able to make up for the function of let-7 when let-7 is abnormal. This research may provide a new view to understand the interaction between miRNAs and genes.

\section{Conclusions}

To the best of our knowledge, this study is the first to demonstrate that let-7 plays a major role in the molting process of Hyalomma asiaticum. Our results suggest that let-7 can regulate the process of molting by modulating the expression of its target ECR in ticks. However, the mechanisms underlying tick development and reproduction need to be further explored.

\section{Abbreviations}

CAAS: Chinese Academy of Agricultural Sciences; DNA: deoxyribonucleic acid; ORF: opening reading frame; miRNA: microRNA; ECR: ecdysteroid receptor; 3'-UTR: 3'-untranslated region; NC: negative control; RT-qPCR: real-time quantitative PCR; RNAi: RNA interference; DLR: dual luciferase reporter assay.
\end{abstract}

\section{Acknowledgements}

Not applicable.

\section{Funding}

This study was financially supported by the National Key Research and Development Program of China (2017YFD0501200, 2016YFC1202000, 2016YFC1202002); the NSFC (31572511); the 973 Program (2015CB150300); ASTIP, FRIP (2014ZL010), CAAS; and NBCIS CARS-38.

\section{Availability of data and materials}

The data supporting the conclusions of this article are included within the article. The HaaECR sequence was submitted to the GenBank database under the accession number MF135614.2.

\section{Authors' contributions}

GYL and JL designed this study and critically revised the manuscript. FW participated in its design and coordination and revised the manuscript. $J \mathrm{~L}, X C L$, JWH, ZGW, JHG and ZQQ participated in sample collection. RHX, ZC, QYR, JXG, QYC, JXL, YH and HW performed the experiments, data analysis and drafted the manuscript. All authors read and approved the final manuscript.
Ethics approval and consent to participate

All protocols were approved by the Animal Research Ethics Committee of the Lanzhou Veterinary Research Institute (permit No. 2009-26).

\section{Consent for publication}

Not applicable.

\section{Competing interests}

The authors declare that they have no competing interests.

\section{Author details}

${ }^{1}$ State Key Laboratory of Veterinary Etiological Biology, Key Laboratory of Veterinary Parasitology of Gansu Province, Lanzhou Veterinary Research Institute, Chinese Academy of Agricultural Science, Xujiaping 1, Lanzhou 730046, Gansu, People's Republic of China. ${ }^{2}$ Gansu Agricultural University, No. 1 Yingmen Village, Anning District, Lanzhou 730070, Gansu, People's Republic of China. ${ }^{3}$ Inspection and Comprehensive Technology Center of Ruili EntryExit Inspection and Quarantine Bureau No. 75, Ruihong Road, Ruili 678600, Yunnan, People's Republic of China. ${ }^{4}$ Jiangsu Co-innovation Center for Prevention and Control of Important Animal Infectious Diseases and Zoonoses, Yangzhou 225009, People's Republic of China. ${ }^{5}$ Department of Engineering, Institute of Biomedical Engineering (IBME), University of Oxford, Oxford OX3 7DQ, UK.

Received: 11 December 2018 Accepted: 6 May 2019

Published online: 15 May 2019

References

1. Brossard M, Wikel SK. Immunology of interactions between ticks and hosts. Med Vet Entomol. 1997;11:270-6.

2. Wikel SK, Bergman D. Tick-host immunology: significant advances and challenging opportunities. Parasitol Today. 1997;13:383-9.

3. Bior AD, Essenberg RC, Sauer JR. Comparison of differentially expressed genes in the salivary glands of male ticks, Amblyomma americanum and Dermacentor andersoni. Insect Biochem Mol Biol. 2002;32:645-55.

4. Goldstein EJC, Thompson C, Spielman A, Krause PJ. Coinfecting deerassociated zoonoses: lyme disease, babesiosis, and ehrlichiosis. Clin Infect Dis. 2001;33:676-85.

5. Stanek G, Wormser GP, Gray J, Strle F. Lyme borreliosis. Lancet. 2012;379:461-73.

6. Carrington JC, Ambros V. Role of microRNAs in plant and animal development. Science. 2003;301:336-8.

7. Smibert P, Lai EC. Lessons from microRNA mutants in worms, flies and mice. Cell Cycle. 2008;7:2500-8.

8. Wienholds E, Plasterk RH. MicroRNA function in animal development. FEBS Lett. 2005;579:5911-22.

9. Hertel J, Bartschat S, Wintsche A, Otto C, Stadler PF. Evolution of the let-7 microRNA family. RNA Biol. 2012;9:231-41.

10. Pasquinelli AE, Reinhart BJ, Slack F, Martindale MQ, Kuroda MI, Maller B, et al. Conservation of the sequence and temporal expression of let-7 heterochronic regulatory RNA. Nature. 2000;408:86-9.

11. Sempere LF, Dubrovsky EB, Dubrovskaya VA, Berger EM, Ambros V. The expression of the let-7 small regulatory RNA is controlled by ecdysone during metamorphosis in Drosophila melanogaster. Dev Biol. 2002:244:170-9.

12. Wu YC, Chen CH, Mercer A, Sokol NS. let-7-Complex microRNAs regulate the temporal identity of Drosophila mushroom body neurons via chinmo. Dev Cell. 2012;23:202-9.

13. Garbuzov A, Tatar M. Hormonal regulation of Drosophila microRNA let-7 and miR-125 that target innate immunity. Fly. 2010;4:306-11.

14. Rubio M, Belles $X$. Subtle roles of microRNAs let-7, miR-100 and miR-125 on wing morphogenesis in hemimetabolan metamorphosis. J. Insect Physiol. 2013;59:1089-94.

15. Chawla G, Sokol NS. Hormonal activation of let-7-C microRNAs via EcR is required for adult Drosophila melanogaster morphology and function. Development. 2012;139:1788-97.

16. Ling L, Ge X, Li Z, Zeng B, Xu J, Aslam AFM, et al. MicroRNA Let-7 regulates molting and metamorphosis in the silkworm, Bombyx mori. Insect Biochem Mol Biol. 2014;53:13-21. 
17. Ashburner M. Sequential gene activation by ecdysone in polytene chromosomes of Drosophila melanogaster. II. The effects of inhibitors of protein synthesis. Dev Biol. 1974;39:141-57.

18. Ashburner M, Richards $G$. Sequential gene activation by ecdysone in polytene chromosomes of Drosophila melanogaster. III. Consequences of ecdysone withdrawal. Dev Biol. 1976;54:241-55.

19. Dubrovsky EB. Hormonal cross talk in insect development. Trends Endocrinol Metab. 2005;16:6-11.

20. Cherbas L, Hu X, Zhimulev I, Belyaeva E, Cherbas P. EcR isoforms in Drosophila: testing tissue-specific requirements by targeted blockade and rescue. Development. 2003;130:271-84.

21. Sempere LF, Sokol NS, Dubrovsky EB, Berger EM, Ambros V. Temporal regulation of microRNA expression in Drosophila melanogaster mediated by hormonal signals and broad-Complex gene activity. Dev Biol. 2003;259:9-18.

22. Tian Z, Zhang P, Luo J, Yin H, Luo J, Xie J, et al. Cloning and characterization of a ribosomal protein $\mathrm{L} 24$ from Hemaphysalis longicornis eggs. Parasitol Res. 2010;107:1213-20.

23. Krüger J, Rehmsmeier M. RNAhybrid: microRNA target prediction easy, fast and flexible. Nucleic Acids Res. 2006:34:W451-4.

24. Rehmsmeier M, Steffen $P$, Höchsmann M, et al. Fast and effective prediction of microRNA/target duplexes. RNA. 2004;10:1507-17.

25. Chen C, Ridzon DA, Broomer AJ, Zhou Z, Lee DH, Nguyen JT, et al. Realtime quantification of microRNAs by stem-loop RT-PCR. Nucleic Acids Res. 2005;33:e179.

26. Hao J, Luo J, Chen Z, Ren Q, Guo J, Liu X, et al. MicroRNA-275 and its target Vitellogenin-2 are crucial in ovary development and blood digestion of Haemaphysalis longicornis. Parasit Vectors. 2017;10:253.

27. Krützfeldt J, Rajewsky N, Braich R, Rajeev KG, TuschI T, Manoharan $M$, et al. Silencing of microRNAs in vivo with 'antagomirs'. Nature. 2005;438:685-9.

28. Guo X, Harmon MA, Laudet V, Mangelsdorf DJ, Palmer MJ. Isolation of a functional ecdysteroid receptor homologue from the ixodid tick Amblyomma americanum (L.). Insect Biochem Mol Biol. 1997;27:945-62.

29. Swevers $L$, Cherbas $L$, Cherbas P, latrou K. Bombyx EcR (BmEcR) and Bombyx USP (BmCF1) combine to form a functional ecdysone receptor. Insect Biochem Mol Biol. 1996;26:217-21.

30. Thomas HE, Stunnenberg HG, Stewart AF. Heterodimerization of the Drosophila ecdysone receptor with retinoid $\mathrm{X}$ receptor and ultraspiracle. Nature. 1993;362:471-5.
31. Yao T-P, Segraves WA, Oro AE, McKeown M, Evans RM. Drosophila ultraspiracle modulates ecdysone receptor function via heterodimer formation. Cell. 1992:71:63-72.

32. Koelle MR, Talbot WS, Segraves WA, Bender MT, Cherbas P, Hogness DS. The Drosophila EcR gene encodes an ecdysone receptor, a new member of the steroid receptor superfamily. Cell. 1991;67:59-77.

33. Lytle JR, Yario TA, Steitz JA. Target mRNAs are repressed as efficiently by microRNA-binding sites in the 5'UTR as in the 3' UTR. Proc Natl Acad Sci USA. 2007:104:9667-72.

34. Ørom UA, Nielsen FC, Lund AH. MicroRNA-10a binds the 5'UTR of ribosomal protein mRNAs and enhances their translation. Mol Cell. 2008;30:460-71.

35. Forman JJ, Legessemiller A, Coller HA. A search for conserved sequences in coding regions reveals that the let-7 microRNA targets Dicer within its coding sequence. Proc Natl Acad Sci USA. 2008;105:14879-84.

36. Carthew RW, Sontheimer EJ. Origins and mechanisms of miRNAs and siRNAs. Cell. 2009;136:642-55.

37. Djuranovic S, Nahvi A, Green R. A parsimonious model for gene regulation by miRNAs. Science. 2011;331:550-3.

38. Nakagawa Y, Henrich VC. Arthropod nuclear receptors and their role in molting. FEBS J. 2009;276:6128-57.

39. Riddiford LM, Hiruma K, Lan Q, Zhou B. Regulation and role of nuclear receptors during larval molting and metamorphosis of Lepidoptera. Am Zool. 1999:39:736-46.

40. Pascual G, Glass CK. Nuclear receptors versus inflammation: mechanisms of transrepression. Trends Endocrinol Metab. 2006;17:321-7.

41. Juan $X$, Chuan-Xing L, Yong-Sheng $L$, Jun-Ying L, Ye M, Ting-Ting S, et al. MiRNA-miRNA synergistic network: construction via co-regulating functional modules and disease miRNA topological features. Nucleic Acids Res. 2011;39:825-36.

42. Xiao $S, M a Y, Z$ hu H, et al. miRNA functional synergistic network analysis of mice with ischemic stroke. Neurol Sci. 2015;36:143-8.

43. Zhao X, Song H, Zuo Z, et al. Identification of miRNA-miRNA synergistic relationships in colorectal cancer. Int J Biol Macromol. 2013;55:98-103.

\section{Publisher's Note}

Springer Nature remains neutral with regard to jurisdictional claims in published maps and institutional affiliations.
Ready to submit your research? Choose BMC and benefit from:

- fast, convenient online submission

- thorough peer review by experienced researchers in your field

- rapid publication on acceptance

- support for research data, including large and complex data types

- gold Open Access which fosters wider collaboration and increased citations

- maximum visibility for your research: over $100 \mathrm{M}$ website views per year

At BMC, research is always in progress.

Learn more biomedcentral.com/submissions 